\title{
The omega-3 fatty acid docosahexaenoic acid favorably modulates the inflammatory pathways and macrophage polarization within aorta of $\mathrm{LDLR}^{-/-}$mice
}

\author{
Cécile Gladine $\cdot$ Monika Zmojdzian $\cdot$ \\ Laurie Joumard-Cubizolles • Marie-Anne Verny • \\ Blandine Comte $\cdot$ Andrzej Mazur
}

Received: 26 May 2014/ Accepted: 25 July 2014/Published online: 19 August 2014

(c) Springer-Verlag Berlin Heidelberg 2014

\begin{abstract}
The omega-3 fatty acid docosahexaenoic acid (DHA) has potent anti-atherogenic properties but its mechanisms of action at the vascular level remain poorly explored. Knowing the broad range of molecular targets of omega-3 fatty acids, microarray analysis was used to openmindedly evaluate the effects of DHA on aorta gene expression in $\mathrm{LDLR}^{-/-}$mice and better understand its local anti-atherogenic action. Mice were fed an atherogenic diet and received daily oral gavages with oils rich in oleic acid or DHA. Bioinformatics analysis of microarray data first identified inflammation and innate immunity as processes the most affected by DHA supplementation within aorta. More precisely, several down-regulated genes were associated with the inflammatory functions of macrophages (e.g., CCL5 and CCR7), cell movement (e.g., ICAM-2, SELP, and PECAM-1), and the major histocompatibility complex (e.g., HLA-DQA1 and HLA-DRB1). Interestingly, several genes were identified as specific biomarkers of macrophage polarization, and their changes suggested a preferential orientation toward a M2 reparative phenotype. This observation was supported by the upstream regulator analysis highlighting the involvement of three main
\end{abstract}

Electronic supplementary material The online version of this article (doi:10.1007/s12263-014-0424-4) contains supplementary material, which is available to authorized users.

C. Gladine $(\bowtie) \cdot$ M. Zmojdzian · L. Joumard-Cubizolles ·

M.-A. Verny - B. Comte · A. Mazur

INRA, UMR1019, UNH, CRNH Auvergne,

Clermont-Ferrand, France

e-mail: cecile.gladine@clermont.inra.fr

C. Gladine - M. Zmojdzian · L. Joumard-Cubizolles ·

M.-A. Verny · B. Comte · A. Mazur

Clermont Université, Université d'Auvergne, Unité de Nutrition

Humaine, Clermont-Ferrand, France regulators of macrophage polarization, namely $\operatorname{PPAR} \gamma$ $\left(z\right.$-score $\left.=2.367, p=1.50 \times 10^{-13}\right)$, INF $\gamma \quad(z$-score $=$ $\left.-2.797, p=2.81 \times 10^{-14}\right)$, and NFKB $(z$-score $=2.360$, $\left.p=6.32 \times 10^{-9}\right)$. Moreover, immunohistological analysis of aortic root revealed an increased abundance of Arg1 $(+111 \%, p=0.01)$, a specific biomarker of M2 macrophage. The present study showed for the first time that DHA supplementation during atherogenesis is associated with protective modulation of inflammation and innate immunity pathways within aorta putatively through the orientation of plaque macrophages toward a M2 reparative phenotype.

Keywords Atherosclerosis - Macrophage polarization Microarray · Omega-3 fatty acid · PUFA - DHA

$\begin{array}{ll}\text { Abbreviations } & \\ \text { ArgI } & \text { Arginase I } \\ \text { B2M } & \beta 2 \text { Microglobulin } \\ \text { CCL5 } & \text { Chemokine (C-C motif) ligand 5 } \\ \text { CCR7 } & \text { C-C chemokine receptor type } 7 \\ \text { CX3CL1 } & \text { Chemokine (C-X3-C motif) ligand 1 } \\ \text { DHA } & \text { Docosahexaenoic acid } \\ \text { EPA } & \text { Eicosapentaenoic acid } \\ \text { FABP4 } & \text { Fatty acid-binding protein } 4 \\ \text { PPAR } \gamma & \text { Peroxisome proliferator-activated receptor } \\ & \text { gamma } \\ \text { HLA } & \text { Human leukocyte } \\ \text { INF } \gamma & \text { Interferon gamma } \\ \text { IPA } & \text { Ingenuity pathway analysis } \\ \text { LCN2 } & \text { Lipocalin 2 } \\ \text { LC- } \omega 3 \text { PUFAs } & \text { Long-chain omega-3 polyunsaturated fatty } \\ & \text { acids } \\ \text { LDLR } & \text { Low-density lipoprotein receptor } \\ \text { NFאB } & \text { Nuclear factor-kappa B }\end{array}$


MHC-II Major histocompatibility complex class II

NCAM1

PECAM1

RANTES

SELP

\section{Neural cell adhesion molecule 1}

Platelet endothelial cell adhesion molecule 1

Regulated on activation, normal $\mathrm{T}$ cell expressed and secreted

Selectin $\mathrm{P}$

\section{Introduction}

Long-chain omega-3 polyunsaturated fatty acids

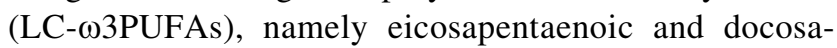
hexaenoic acids (EPA and DHA), have recognized antiatherogenic properties (De Caterina and Zampolli 2006). These have been mainly attributed to their hypolipidemic effect but several lines of evidence show that LCw3PUFAs can reduce atherosclerosis independently of the systemic risk factors, suggesting direct anti-atherogenic action of LC- $\omega 3$ PUFAs at the vascular level (Ishikado et al. 2013). However, the cellular and molecular targets of LC- $\omega 3$ PUFAs within blood vessels remain largely unexplored. In fact, investigations of the vascular effects of LC- $\omega 3$ PUFAs have so far exclusively used targeted approaches providing a partial view of their spectrum of action. Indeed, several in vivo and in vitro studies in other tissues have shown that LC- $\omega 3$ PUFAs have a very large panel of cellular and molecular targets leading to the modulation of the expression of many genes (Knoch et al. 2009; Gladine et al. 2012; Jump et al. 2012).

It is well recognized that atherosclerotic plaque initiation and progression are strongly dependent on the recruitment and activity of innate immune cells, such as monocytes/macrophages, dendritic cells, and mast cells. More recently, monocytes/macrophages came to the forefront of research owing to new awareness that they may represent a more heterogeneous and phenotypically plastic population than initially believed. Indeed, macrophages are now described as a heterogeneous population of cells showing adaption capacity in response to a variety of microenvironmental signals, and their phenotypic polarization is believed to play a key role in the fate of atherosclerotic plaques (Shalhoub et al. 2011). Actually, recent evidence suggests that various stages of atherogenesis are associated with distinct macrophage subtypes (Pello et al. 2011). These mainly include the classically activated (M1) macrophages, abundant in advanced lesion and exhibiting potent inflammatory properties, and the alternatively activated (M2) population involved in the early stages of atherogenesis and having reparative properties (KhallouLaschet et al. 2010).

\section{Materials and methods}

Mice, diets, and study outline

The aim of the present experiment was to investigate the anti-atherogenic effects of DHA at the vascular level. Since wild-type mice do no develop atherosclerosis (regardless of strains or diets), no wild-type control mice were included in the study and $\mathrm{LDLR}^{-1-}$ mice were chosen. In this animal model, atherogenesis is induced by nutritional intervention and lesions are very similar to humans (Ishibashi et al. 1994). The mice were purchased from Jackson Laboratories (Charles River Laboratories, L'Arbresle, France), housed 10 per cage, in a temperature-controlled environment $\left(22 \pm 0.8{ }^{\circ} \mathrm{C}\right)$ with a 12-h light-dark cycle, and allowed free access to food and water. At 8 weeks of age, mice were given a diet enriched in animal fat $(10 \%$ of lard) and low in cholesterol $(0.045 \%$, Sigma-Aldrich C75209), and were randomized into two different groups ( $n=8$ /group) on the basis of body weight. In parallel to the diet, mice received by daily oral gavages $(50 \mu \mathrm{L}$, 5 days/week) either oleic acid-rich sunflower oil (Lesieur, Asnières-sur-Seine, France; control group) or a mixture of oleic acid-rich sunflower oil and DHA-rich tuna oil (OMEGAVIE $^{\circledR}$ DHA 90 TG, Polaris Nutritional Lipids, France, containing $90 \%$ of DHA as TG) providing $2 \%$ (or $35.5 \mathrm{mg} / \mathrm{d} / \mathrm{mouse}$ ) of energy as DHA (DHA group). After 20 weeks of feeding, mice were anaesthetized $(40 \mathrm{mg}$ pentobarbital $/ \mathrm{kg}$ body weight). After rapid washing with sterilized PBS, heart and aorta samples were immediately 
frozen into liquid nitrogen and stored at $-80{ }^{\circ} \mathrm{C}$ until further analyses.

\section{Microarray analysis}

The entire aorta (i.e., thoracic and abdominal sections) was used for RNA extraction. RNA was extracted using the RNeasy Plus Micro Kit (Qiagen, Hilden, Germany) according to the manufacturer's instructions. Purity and yield of RNA was assessed using the NanoDrop 1000 spectrophotometer (Thermo Scientific, Wilmington, DE, USA). Extracted RNA was then amplified and labeled using the Agilent Low-Input QuickAmp labeling kit (51902306, Agilent technologies, Wilmington, DE, USA) according to the manufacturer's instructions. Purity and fluorescent intensity of the amplified and labeled cRNA was assessed using the NanoDrop 1000 spectrophotometer (Thermo Scientific, Wilmington, DE, USA): all samples had a yield of $825 \mathrm{ng}$ cRNA and a specific activity of 8.0 pmol $\mathrm{Cy} 3$ or $\mathrm{Cy} 5$ per mg cRNA. The fluorescently labeled cRNAs were hybridized using the Agilent gene expression hybridization kit (5188-5242, Agilent technologies, Wilmington, DE, USA). Briefly, $825 \mathrm{ng}$ cyanine 3-labeled, linearly amplified cRNA was mixed with $825 \mathrm{ng}$ cyanine 5-labeled, linearly amplified cRNA. The mix was loaded onto the Agilent's Mouse GE $4 \times 44 \mathrm{~K}$ v2 Microarrays (Agilent Microarray Design ID 026655) following a reference design. Hybridization proceeded in a hybridization oven set to $65^{\circ} \mathrm{C}$ for $17 \mathrm{~h}$. Then, the slides were washed in solutions I, II, and III (Agilent technologies, Wilmington, DE, USA) and air-dried.

Slides were scanned immediately after washing using the Agilent Microarray Scanner (G2505 B, Agilent technologies, Wilmington, DE, USA), and spot identification and quantification were performed using Agilent Feature Extraction Software 10.7 (Agilent technologies, Wilmington, DE, USA). The complete formatted dataset was deposited as Gene Expression Omnibus accession GSE59661, which can be accessed at http://www.ncbi.nlm.nih.gov/geo/.

Ratios were filtered in accordance with their variability among the eight comparisons, and genes with high variability were removed from the analysis. Statistical analyses were performed using the free R 2.1 software (http://www. r-project.org). The log ratio between experimental and control samples was analyzed with Student's $t$ test to detect differentially expressed genes, and probability values were adjusted using the Bonferroni correction for multiple testing at $1 \%$ to eliminate false positives. Genes selected by these criteria are referred to as "differentially expressed genes".

To validate microarray data, real-time quantitative PCR was performed on the same RNA sample for a subset of genes identified as differentially expressed (IGFBP2,
FABP4, CCL5, FBLN7, RBP7). High-Capacity cDNA Reverse Transcription Kit (Applied Biosystems, CA, USA) was used to reverse transcribe RNA to cDNA. The primers were identified using Primer Express software (Applied Biosystems, CA, USA) (Supporting Information, Table S1). The qRT-PCR was carried out on Applied Biosystems Prism 7900HT system using the Power SYBR Green PCR Master Mix kit (Applied Biosystems, CA, USA). After an initial denaturation of $10 \mathrm{~min}$ at $95^{\circ} \mathrm{C}$, a two-step cycling conditions were as follows: 15 -s denaturation at $95{ }^{\circ} \mathrm{C}$ and annealing/extension at $60{ }^{\circ} \mathrm{C}$ for $60 \mathrm{~s}$, cycled 40 times. The expression levels were calculated using the $\Delta \Delta \mathrm{Ct}$ method (Livak and Schmittgen 2001).

Gene expression data analysis

\section{Functional enrichment analysis}

Functional analysis was performed to identify biological functions and/or diseases that were most significant to our dataset. Right-tailed Fisher's exact test was used to calculate a significant $P$ value for each functional category as referenced in Ingenuity ${ }^{\circledR}$ Knowledge Base. Downstream effects analysis is based on the expected causal effects between genes and functions; these expected effects are derived from the literature compiled in the Ingenuity ${ }^{\circledR}$ Knowledge Base. The analysis examines genes in the dataset that are known to affect functions, compares the genes' direction of change to expectations derived from the literature, and then issues a prediction for each function based on the direction of change. The direction of change is the gene expression in the DHA group relative to the control group. IPA uses the regulation $z$-score algorithm to make predictions. The $z$-score algorithm is designed to reduce the chance that random data will generate significant predictions. When the $z$-score is positive, IPA predicts that the biological process or disease is trending toward an increase, whereas when the $z$-score is negative, IPA predicts that the biological process or disease is trending toward a decrease. A $z$-score $\geq 2$ or $\leq-2$ indicates that the function is statistically significantly increased or decreased.

\section{Canonical pathway analysis}

Lists of mRNAs differentially expressed between the control and the DHA groups were uploaded in the IPA tool (Ingenuity ${ }^{\circledR}$ Systems, www. ingenuity.com) and analyzed based on the IPA library of canonical pathways. The significance of the association between each list and a canonical pathway was measured by Fisher's exact test. As a result, a $p$ value was obtained, determining the probability that the association between the genes in our dataset and a canonical pathway can be explained by chance alone. 


\section{Upstream regulator analysis}

The goal of the IPA upstream regulator analysis was to identify the cascade of upstream transcriptional regulators that can explain the observed gene expression changes in the dataset. The upstream regulator analysis is based on prior knowledge of expected effects between transcriptional regulators and their target genes stored in the Ingenuity ${ }^{\circledR}$ Knowledge Base. The analysis examines how many known targets of each transcription regulator are present in the user's dataset and also compares their direction of change (i.e., expression in the DHA group relative to the control group) to what is expected from the literature in order to predict likely relevant transcriptional regulators. If the observed direction of change is mostly consistent with a particular activation state of the transcriptional regulator ("activated" or "inhibited"), then a prediction is made about that activation state. For each potential transcriptional regulator ("TR"), two statistical measures, an overlap $p$ value and an activation $z$-score, are computed. The overlap $p$ value calls likely upstream regulators based on significant overlap between dataset genes and known targets regulated by a TR. The activation $z$-score is used to infer likely activation states of upstream regulators based on comparison with a model that assigns random regulation directions.

\section{Immunohistochemical analysis}

Cryosections (10 $\mu \mathrm{M}, 4$ sections/slides, and $8 \mathrm{mice} /$ group) were performed at the level of aortic root (i.e., the aortic valve from its position at the left ventricular outlet to its junction with the ascending portion of the aorta). After drying for $15 \mathrm{~min}$ at room temperature, cryosections were fixed in $70 \%$ ethanol and cold acetone $\left(4{ }^{\circ} \mathrm{C}\right)$. After a rapid washing in PBS, sections were then incubated overnight with the primary antibodies: rat anti-mouse Mac3 monoclonal antibody (BD Biosciences, Le Pont de Claix, France) and rabbit anti-mouse ArgI polyclonal antibody (GeneTex, Irvine, CA, USA). The staining was revealed by using fluorescent secondary antibodies: cyanine 3 goat anti-rat $\operatorname{IgG}(\mathrm{H}+\mathrm{L})$ for Mac3 (Invitrogen, Cergy Pontoise, France) and fluorescein isothiocyanate (FITC) goat anti-rabbit (GeneTex) for ArgI. Immunostained slides were cover-mounted with Fluoromont G (Southern Biotech, Birmingham, AL, USA). The fluorescence was detected with a Zeiss Axioplan $2^{\mathrm{E}}$ microscope equipped with the AxioCam MRm camera and the AxioVisionRel.4.8 image capture software. Images were acquired using the EC "Plan-Neofluar" $40 \times / 0.75$ objective of the microscope. Three random fields were analyzed on each section, and the surface of each lesion was delimited based on the staining of Mac3 using the ROI Manager tool in the ImageJ free software (http://rsb.info.nih.gov/ij/). The intensity of each staining (i.e., Mac3 and ArgI) is relative to this delimited surface. Differences between the mean intensities of control and DHA mice were analyzed using a Student's $t$ test and considered significant at $p<0.05$.

\section{Results}

Aorta gene expression by microarray analysis

\section{Differentially expressed genes}

Overall gene expression profiling of aorta was carried out using microarray analysis in order to determine the major functional targets of DHA at the vascular level and to gain insight into the mechanisms of action underlying anti-atherogenic effects of DHA. As shown in Fig. 1, among the 24,241 genes (i.e., 39,429 transcripts) represented in the Mouse GR $4 \times 44$ k v2 microarray, 532 genes were found to be differentially expressed $(p<0.01)$ between the control and DHA groups, with $58 \%$ of genes being up-regulated and $42 \%$ being down-regulated by DHA supplementation. Real-time quantitative PCR was performed using same RNA samples on a subset of genes identified as differentially expressed in aorta. For all the genes studied, the expression values were concordant with microarray data (Supporting Information, Figure S1).

\section{Functional enrichment and canonical pathway analyses}

To better understand which and how biological processes have been affected by differentially regulated mRNAs, functional annotations and canonical pathway analysis were performed in IPA (Fig. 2). Functional annotations allowed

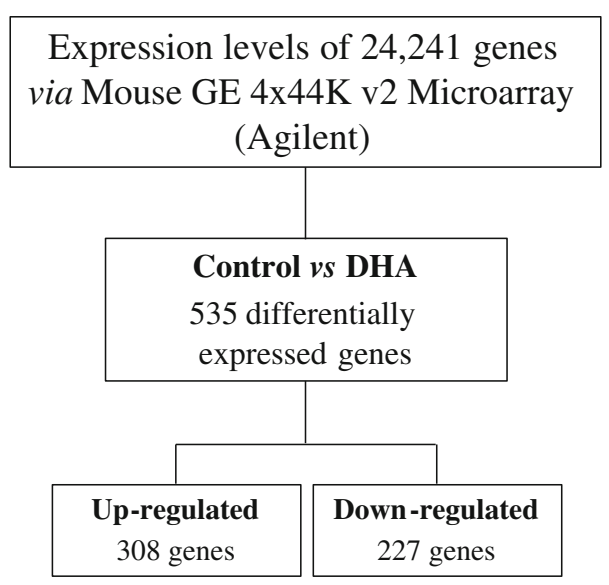

Fig. 1 Flowchart of the number of genes differentially expressed in the aorta of control versus DHA mice 
determining the 5 biological functions that were most significant to our dataset (Fig. 2a). Further enrichment analysis on defined (canonical) pathways of IPA knowledge base provided the list of the 5 most significant overrepresented pathways (Fig. 2b). Interestingly, both types of analyses (i.e., functional annotation and canonical pathways) highlighted the overrepresentation of inflammatory processes and innate immunity. Indeed, 2 of the top 5 biological functions were identified as Hematological System Development and Function and Immune Cell Trafficking, and the top five canonical pathways were all directly or indirectly related to antigen presentation. The Hematological System Development and Function (70 sub-functions) and Immune Cell Trafficking (51 sub-functions) shared most of their subfunctions (i.e., 50 common sub-functions). Moreover, the 14 significantly altered sub-functions (i.e., $-2 \geq z$-score $\geq 2$ ) were exclusively associated with immune cell movement and, most importantly, exclusively decreased (Table 1). The analysis of the genes associated with these sub-functions showed a remarkable number of down-regulated genes related to the inflammatory function of macrophages (Fig. 3). These include the chemokines CCL5 (FC = -1.49), CXCL12 (FC $=-1.29)$, CXCL11 $(\mathrm{FC}=1.49)$, PF4 $(\mathrm{FC}=1.65)$, and the chemokine receptor CCR7 $(\mathrm{FC}=-1.43)$ as well as the pro-inflammatory mediator FN1 (FC $=-1.63)$. A second set of overrepresented molecules are related to cell-to-cell interaction and include the adhesion molecules ICAM2 $(\mathrm{FC}=-1.34)$, SELP
$(\mathrm{FC}=-1.30)$, PECAM1 $(\mathrm{FC}=-1.29)$, the transmembrane chemokine CX3CL1 ( $\mathrm{FC}=-1.12)$ as well as the extracellular protein THBS2 ( $\mathrm{FC}=-1.29)$. Interestingly, CCL5 and CCR7 together with the fatty acid transporter FABP4 $(\mathrm{FC}=+2.96)$ and the coagulation factor F13A1 $(\mathrm{FC}=+1.65)$ are recognized biomarkers of macrophage polarization. Moreover, the direction of changes suggests a preferential orientation toward the reparative M2 phenotype. The analysis of the genes associated with the top five canonical pathways (Table 2) also supports this indication. Indeed, most genes identified belong to the major histocompatibility complex (i.e., B2 M, CD74, HLA-B, HLA-C, HLA-DQA1, HLA-DRB1, HLA-DQB1, and H2-Q8), which is known to be up-regulated in pro-inflammatory M1 macrophages. These genes together with lipocalin 2 (LCN2, $\mathrm{FC}=-1.47)$, another biomarker of M1 macrophage, were also associated with the functional annotation analysis (Supporting Information, Table S2).

\section{Upstream regulator analysis}

The IPA "upstream analysis" was run to identify the cascade of upstream transcriptional regulators that can explain the observed gene expression changes. The upstream regulator analysis is based on prior knowledge of expected effects between transcriptional regulators and their target genes stored in the Ingenuity ${ }^{\circledR}$ Knowledge Base. It examines how many known targets of each transcription

a Top 5 biological functions

a Top 5 biological functions
Cardiovascular System Development and Function
Organismal Development
Tissue Development
Hematological System Development and Function
Immune Cell Tafficking

b Top 5 canonical pathways

Antigen Presentation Pathway

OX40 Signaling pathway

Cytotoxic T Lymphocyte-mediated Apoptosis of Target Cells

Allograft Rejection Signaling

Crosstalk between Dendritic Cells and Natural Killer Cells
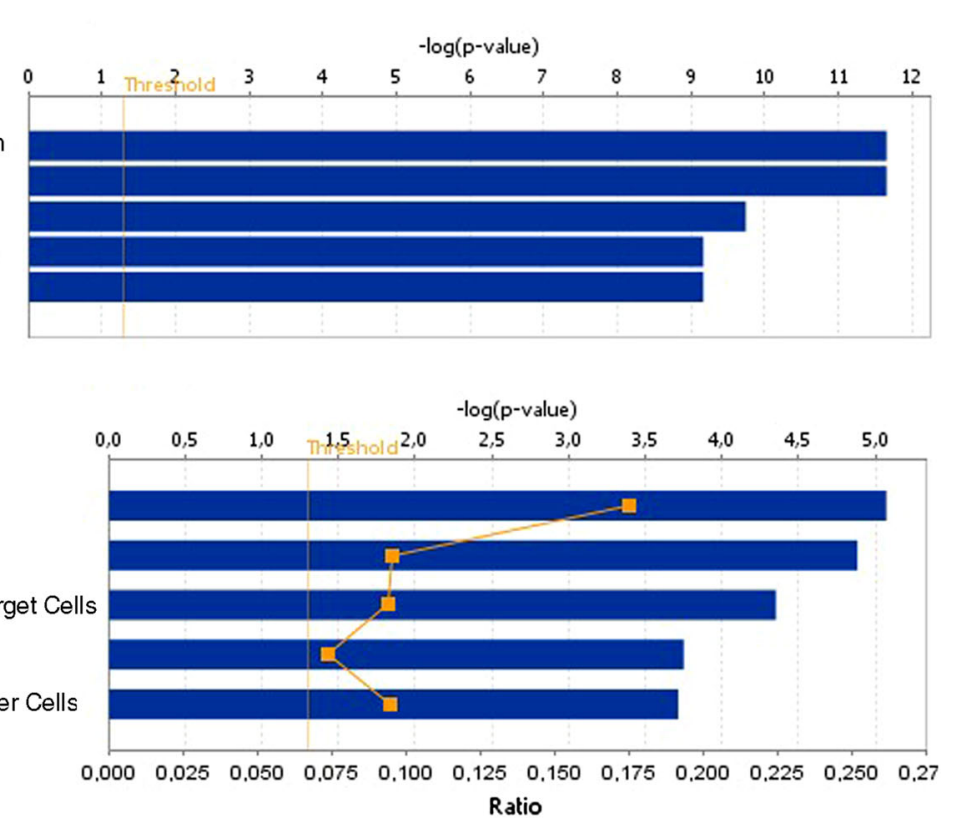

Fig. 2 Most significant biological processes affected in the aorta by DHA supplementation in LDLR $^{-1-}$ mice. The list of genes differentially expressed between control and DHA mice was uploaded in the IPA tool (Ingenuity ${ }^{\circledR}$ System, www.ingenuity.com) and analyzed based on the Ingenuity ${ }^{\circledR}$ Knowledge Base. The significance of the association between the genes list and the function or pathway ( $p$ value) was measured using the right-tailed Fisher's exact test. "Ratio" indicates the number of genes in a given pathway divided by the total number of genes making up the pathway 
Table 1 Significantly altered sub-functions. Ingenuity systems found 70 sub-functions in the Hematological system development function and 51 sub-functions in the Immune cell trafficking function. Fourteen sub-functions were found to be significantly decreased (i.e., $z$-score $\leq-2$ )

\begin{tabular}{ll}
\hline Sub-functions annotation & Activation $z$-score \\
\hline Cell movement of myeloid cells & -2.87 \\
Cell movement of leukocytes & -2.58 \\
Cell movement of peripheral blood leukocytes & -2.58 \\
Cell movement of granulocytes & -2.54 \\
Cell movement of PBMCs & -2.39 \\
Cell movement of mononuclear leukocytes & -2.31 \\
Cell movement of phagocytes & -2.26 \\
Lymphocyte migration & -2.24 \\
Chemotaxis of mononuclear leukocytes & -2.22 \\
Chemotaxis of phagocytes & -2.20 \\
Homing of mononuclear leukocytes & -2.20 \\
Chemotaxis of myeloid cells & -2.12 \\
Chemotaxis of leukocytes & -2.02 \\
Homing of leukocytes & -2.00 \\
\hline
\end{tabular}

${ }^{\text {a IPA downstream effects analysis identifies functions that are }}$ expected to increase or decrease, given the observed gene expression changes the experimental dataset. Downstream effects analysis is based on the expected causal effects between genes and functions; the expected causal effects are derived from the literature compiled in the Ingenuity ${ }^{\circledR}$ Knowledge Base. The analysis examines genes in the dataset that are known to affect functions, compares the genes' direction of change to expectations derived from the literature, and then issues a prediction for each function based on the direction of change. IPA uses the regulation $z$-score algorithm to make predictions. The $z$-score algorithm is designed to reduce the chance that random data will generate significant predictions. A negative $z$-score $\leq-2$ means that the sub-function is statistically significantly decreased

regulator are present in the dataset and also compare their direction of change to what is expected from the literature in order to predict likely relevant transcriptional regulators. As shown in Table 3, the analysis of our dataset revealed significant modulations (activation $z$-score $\geq 2$ or $\leq-2$ ) of 20 transcriptional regulators, 7 being activated (Table 3A) and 13 being inhibited (Table 3B). Interestingly, the activated transcriptional regulators were mostly related to the peroxisome proliferator-activated receptors (PPARs)dependent signaling pathways with PPAR $\gamma$ being the most significant activated upstream regulator associated with our dataset $\left(z\right.$-score $\left.=2.367, p=1.50 \times 10^{-13}\right)$. Concerning the inhibited upstream regulators, they include several members of the interferon (INF) family with INF $\gamma$ being the most significant inhibited regulator $(z$-score $=-2.797$, $\left.p=2.81 \times 10^{-14}\right)$. The well-known inflammatory regulator nuclear factor-kappa $\mathrm{B}(\mathrm{NF \kappa B})$ was also significantly associated with our dataset $(z$-score $=-2.360$, $p=6.32 \times 10^{-9}$ ). It should be noticed that PPAR $\gamma, \mathrm{INF} \gamma$, and $\mathrm{NF \kappa B}$ are three main transcriptional regulators involved in macrophage polarization. Moreover, the analysis of the targets of these 3 most significantly modulated upstream regulators (Fig. 4 and Supporting Information, Table S3) reveals that almost all the macrophage polarization biomarkers (except F13A1) previously identified (i.e., CCL5, CCR7, FABP4, LCN2, B2 M, CD74, HLADQA1, HLA-DRB1, and HLA-DQB1) are directly or indirectly regulated by PPAR $\gamma, \mathrm{INF} \gamma$, and/or NFKB. Most of the genes involved in cell-to-cell interaction ion our dataset (i.e., PECAM1, SELP, CX3CL1, and NCAM1) also belong to the list of the target genes of INF $\gamma$ and NFKB.

Immunohistological analysis of atherosclerotic lesions

We have previously shown that DHA supplementation reduced plaque extent $(-35 \%, p<0.001)$ in aortic root cryosections from these $\mathrm{LDLR}^{-1-}$ mice (Gladine et al. 2014). This is consistent with the representations of plaque size reported in Fig. 5a (top 2 pictures) showing smaller plaques in the DHA group in comparison with the control group. The immunofluorescent analysis aimed to get further insights into the impact of DHA on plaque composition, notably regarding macrophage infiltration and phenotype. Two biomarkers, namely Mac3 and ArgI, were selected to identify macrophages $\left(\mathrm{Mac}^{+}\right)$and reparative M2 macrophages $\left(\mathrm{ArgI}^{+}\right)$, respectively. Intensities of staining calculated for each marker (Fig. 5b, c) are relative to the lesion surface as delimited by the Mac3 staining.

Concerning macrophages infiltration in the lesion (Mac $3^{+}$cells), the overall surface stained was higher in control mice (Fig. 5a, middle 2 pictures), which is consistent with their larger lesions in comparison with DHA mice. However, when calculating the staining intensity relative to the lesion surface, it should be noted that DHA supplementation was associated with a higher macrophage intensity in the lesion $(+130 \%, p=0.01)$ in comparison with control mice (Fig. 5b). But, interestingly, when looking at the macrophages subtype as determined by the distinct expression of the arginase $\mathrm{I}$ isoform (Fig. 5a, 2 bottom pictures), lesions of DHA-supplemented mice were characterized by a higher intensity of type M2 macrophages $\left(\mathrm{ArgI}^{+}\right.$cells, $+175 \%, p=0.001$ ) (Fig. 5c). Overall, these results suggest that lesion of mice given DHA was rich in macrophages but these macrophages were mainly oriented toward a M2 reparative phenotype which reinforced the interpretation of the gene expression data.

\section{Discussion}

Using an integrated approach combining in vivo experiment, microarray analysis, and immunohistochemical 


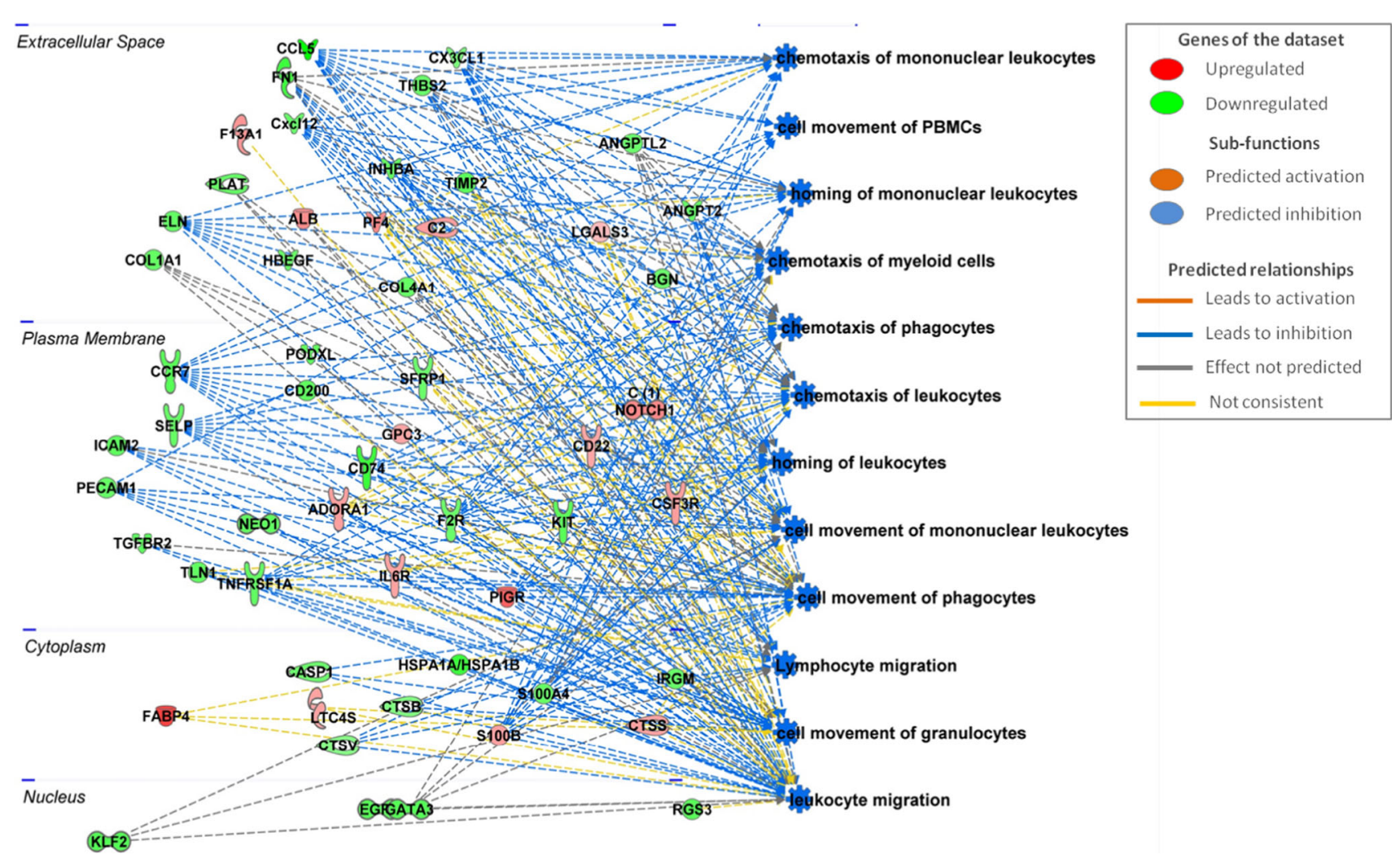

Fig. 3 Network diagram showing the genes associated with the significantly altered sub-functions related to "Hematological system development" and "Immune cell trafficking" functions. Ingenuity systems found 55 genes of the dataset associated with the significantly decreased sub-functions identification of specific macrophages biomarkers, our results show for the first time that 1) DHA supplementation during atherogenesis is associated with protective modulations of inflammation and innate immunity within aorta, and 2) DHA induced changes in the expression of several referenced biomarkers of macrophage polarization, suggesting a preferential orientation of plaque macrophages toward a M2 reparative phenotype.

Molecular targets of LC $\omega 3$-PUFA at the vascular level have been poorly investigated so far and using exclusively targeted approaches (Matsumoto et al. 2008), which is open to criticism knowing the broad spectrum of action of LC 63 -PUFA and/or their metabolites (Jump et al. 2012). The present nutrigenomic study shows that pathways related to inflammation and innate immunity at the vascular level are favorably influenced by DHA. This is consistent with the well-known anti-inflammatory properties of LC 133 -PUFA (Calder 2012) and a transcriptomic study conducted on human blood cells reporting that DHA suppressed the effects of LPS on the expression of genes related to immune defense and inflammatory response (Dawson et al. 2012). Most importantly, our study allowed the identification of several genomic targets of DHA within aorta. These notably include CCL5, CCR7, and FN1, three mediators of inflammation-promoting immune cells or fibroblasts recruitment to inflammatory sites including atherosclerotic plaques (Eriksson 2004; Keophiphath et al. 2010; Moore et al. 2013). Indeed, CCL5, also known as RANTES, is a chemokine secreted by fibroblasts, platelets, and monocytes/macrophages triggering monocytes arrests in atherosclerotic arteries (Huo et al. 2000; Eriksson 2004). The role of CCR7 in atherogenesis is more controversial since CCR7 knockout experiments showed both atherosclerosis regression (Luchtefeld et al. 2010) and progression (Wan et al. 2013). Nevertheless, CCR7 is crucially involved in several fundamental processes shaping the structural and functional organization of the adaptive immune system (Forster et al. 1999). Therefore, the decreased abundance of CCR7 mRNA induced by DHA supplementation may reflect a moderation of the adaptive immune system, which could be beneficial in the condition of advanced atherosclerotic plaque. Fibronectin (FN1) is a glycoprotein of the extracellular matrix produced by different cell types including activated macrophages and playing a chemoattractant role (Tsukamoto et al. 1981). These three 
Table 2 Genes associated with the top 5 canonical pathways. Lists of mRNAs differentially expressed between the control and the DHA groups was uploaded in the IPA tool (Ingenuity ${ }^{\circledR}$ Systems, www.ingenuity.com) and analyzed based on the IPA library of canonical pathways. The list of differentially expressed genes associated with each pathway is reported below

\begin{tabular}{|c|c|c|c|c|}
\hline Gene symbol & Gene name & Entrez gene & Fold change & Type(s) \\
\hline \multicolumn{5}{|c|}{ Antigen-presenting pathway } \\
\hline B2 M & Beta-2-microglobulin & 12,010 & -1.301 & Transmembrane receptor \\
\hline $\mathrm{CD} 74$ & CD74 molecule. major histocompatibility complex. class II & 16,149 & -1.671 & Transmembrane receptor \\
\hline HLA-B & - & 15,018 & -1.291 & Transmembrane receptor \\
\hline HLA-C & Major histocompatibility complex. class I. C & 14,972 & -1.240 & Other \\
\hline HLA-DQA1 & Major histocompatibility complex. class II. DQ alpha 1 & 14,960 & -1.499 & Transmembrane receptor \\
\hline HLA-DRB1 & Major histocompatibility complex. class II. DR beta 1 & 14,969 & -1.690 & Transmembrane receptor \\
\hline PSMB9 & Proteasome (prosome. macropain) subunit. beta type. 9 & 16,912 & -1.294 & Peptidase \\
\hline \multicolumn{5}{|c|}{ Ox40 Signaling pathway } \\
\hline B2 M & Beta-2-microglobulin & 12,010 & -1.301 & Transmembrane receptor \\
\hline BCL2L1 & BCL2-like 1 & 12,048 & 1.493 & Other \\
\hline H2-Q8 & Histocompatibility 2. Q region locus 8 & 15,019 & -1.431 & Other \\
\hline HLA-B & - & 15,018 & -1.291 & Transmembrane receptor \\
\hline HLA-C & Major histocompatibility complex. class I. C & 14,972 & -1.240 & Other \\
\hline HLA-DQA1 & Major histocompatibility complex. class II. DQ alpha 1 & 14,960 & -1.499 & Transmembrane receptor \\
\hline HLA-DQB1 & Major histocompatibility complex. class II. DQ beta 1 & 14,961 & -1.675 & Other \\
\hline HLA-DRB1 & Major histocompatibility complex. class II. DR beta 1 & 14,969 & -1.690 & Transmembrane receptor \\
\hline JUN & Jun proto-oncogene & 16,476 & -1.525 & Transcription regulator \\
\hline \multicolumn{5}{|c|}{ Cytotoxic T lymphocyte-mediated apoptosis of target cells } \\
\hline B2M & Beta-2-microglobulin & 12,010 & -1.301 & Transmembrane receptor \\
\hline DFFA & DNA fragmentation factor. $45 \mathrm{kDa}$. alpha polypeptide & 13,347 & 1.461 & Enzyme \\
\hline H2-Q8 & Histocompatibility 2. Q region locus 8 & 15,019 & -1.431 & Other \\
\hline HLA-B & - & 15,018 & -1.291 & Transmembrane receptor \\
\hline HLA-C & Major histocompatibility complex. class I. C & 14,972 & -1.240 & Other \\
\hline HLA-DQA1 & Major histocompatibility complex. class II. DQ alpha 1 & 14,960 & -1.499 & Transmembrane receptor \\
\hline HLA-DQB1 & Major histocompatibility complex. class II. DQ beta 1 & 14,961 & -1.675 & Other \\
\hline HLA-DRB1 & Major histocompatibility complex. class II. DR beta 1 & 14,969 & -1.690 & Transmembrane receptor \\
\hline \multicolumn{5}{|c|}{ Allograft rejection signaling } \\
\hline B2 M & Beta-2-microglobulin & 12,010 & -1.301 & Transmembrane receptor \\
\hline H2-Q8 & Histocompatibility 2. Q region locus 8 & 15,019 & -1.431 & other \\
\hline HLA-B & - & 15,018 & -1.291 & Transmembrane receptor \\
\hline HLA-C & Major histocompatibility complex. class I. C & 14,972 & -1.240 & Other \\
\hline HLA-DQA1 & Major histocompatibility complex. class II. DQ alpha 1 & 14,960 & -1.499 & Transmembrane receptor \\
\hline HLA-DQB1 & Major histocompatibility complex. class II. DQ beta 1 & 14,961 & -1.675 & Other \\
\hline HLA-DRB1 & Major histocompatibility complex. class II. DR beta 1 & 14,969 & -1.690 & Transmembrane receptor \\
\hline \multicolumn{5}{|c|}{ Cross talk between dendritic cells and natural killer cells } \\
\hline ACTA1 & Actin. alpha 1. skeletal muscle & 11,459 & -1.314 & Other \\
\hline ACTG1 & Actin. gamma 1 & 11,465 & -1.315 & Other \\
\hline ACTG2 & Actin. gamma 2. smooth muscle. enteric & 11,468 & -1.394 & Other \\
\hline CCR7 & Chemokine $(\mathrm{C}-\mathrm{C}$ motif) receptor 7 & 12,775 & -1.425 & G-protein-coupled receptor \\
\hline HLA-B & - & 15,018 & -1.291 & Transmembrane receptor \\
\hline HLA-C & Major histocompatibility complex. class I. C & 14,972 & -1.240 & Other \\
\hline HLA-DRB1 & Major histocompatibility complex. class II. DR beta 1 & 14,969 & -1.690 & Transmembrane receptor \\
\hline IL2RG & Interleukin 2 receptor. gamma & 16,186 & -1.253 & Transmembrane receptor \\
\hline TLN1 & Talin 1 & 21,894 & -1.324 & Other \\
\hline
\end{tabular}


Table 3 Most significant upstream regulators associated with the dataset

\begin{tabular}{lcc}
\hline Upstream regulator & Activation $z$-score & $p$ value of \\
\hline (a) List of the most significant activated upstream regulators \\
PPARG & 2.367 & $1.50 \mathrm{E}-13$ \\
PPARA & 2.384 & $6.45 \mathrm{E}-10$ \\
PPARD & 3.160 & $1.56 \mathrm{E}-08$ \\
estrogen receptor & 2.688 & $2.50 \mathrm{E}-07$ \\
PPAR $\alpha$-RXR $\alpha$ & 2.000 & $4.42 \mathrm{E}-04$ \\
RXRA & 2.068 & $7.05 \mathrm{E}-03$ \\
ESRRA & 2.414 & $8.68 \mathrm{E}-02$
\end{tabular}

(b) List of the most significant inhibited upstream regulators

$\begin{array}{lll}\text { IFNG } & -2.797 & 2.81 \mathrm{E}-14 \\ \text { NFkB (complex) } & -2.360 & 6.32 \mathrm{E}-09 \\ \text { PRL } & -2.153 & 1.07 \mathrm{E}-08 \\ \text { IL6 } & -2.226 & 7.88 \mathrm{E}-07 \\ \text { IL27 } & -2.613 & 1.26 \mathrm{E}-06 \\ \text { Pdgf (complex) } & -2.574 & 4.99 \mathrm{E}-05 \\ \text { PDGF BB } & -2.009 & 1.40 \mathrm{E}-04 \\ \text { TCR } & -2.416 & 1.75 \mathrm{E}-04 \\ \text { Interferon alpha } & -2.771 & 4.23 \mathrm{E}-04 \\ \text { IFN alpha/beta } & -2.156 & 7.53 \mathrm{E}-04 \\ \text { CCL13 } & -2.425 & 2.30 \mathrm{E}-03 \\ \text { EBI3 } & -2.177 & 3.55 \mathrm{E}-03 \\ \text { EDN1 } & -2.672 & 3.74 \mathrm{E}-03\end{array}$

The upstream regulator analysis is based on prior knowledge of expected effects between transcriptional regulators and their target genes stored in the Ingenuity ${ }^{\circledR}$ Knowledge Base. For each upstream regulator, the $p$ value of overlap calls likely upstream regulators based on significant overlap between dataset genes and known targets regulated by a transcriptional regulator. The activation $z$-score is used to infer likely activation states of upstream regulators based on comparison with a model that assigns random regulation directions

specific proteins (i.e., CCL5, CCR7, and FN1) have never been identified before as target genes of DHA. Our results are nevertheless in accordance and complementary with previous results in vitro showing that LC- $\omega 3$ PUFAs reduce the production of chemoattractants by immune cells (Calder 2013), which is probably one of the primary anti-atherosclerotic actions of DHA at the vascular level. Another set of genes of interest related to cell-to-cell interaction were also associated with DHA supplementation in our model. These include the adhesion molecules ICAM2, SELP, PECAM1, the transmembrane chemokine CX3CL1 as well as the extracellular protein THBS2 that were all down-regulated in the aorta of DHA-supplemented mice. These proteins are produced by endothelial cells, monocytes/macrophages, and/or platelets (Jaffe et al. 1985) and contribute to monocyte adhesion to endothelial cell, a critical step in the initiation and progression of atherosclerosis (Galkina and Ley 2007;
Zernecke et al. 2008). Whereas these specific proteins have not been all identified previously, several in vitro and ex vivo experiments have reported decreased expression of adhesion molecules on the surface of monocytes, macrophages, or endothelial cells following exposure to LC- $\omega 3$ PUFAs (De Caterina et al. 1994; Weber et al. 1995; Collie-Duguid and Wahle 1996; Hughes et al. 1996; Miles et al. 2000). Finally, many down-regulated genes overrepresented in our dataset belong to the major histocompatibility complex class II (i.e., HLA-DQA1, HLA-DRB1, and HLA-DQB1). Molecules from the major histocompatibility complex class II (MHC-II) play a critical role in the induction of immune responses by presenting peptides of foreign antigens to $\mathrm{CD} 4+\mathrm{T}$ lymphocytes, which results in their activation and proliferation (Reith and Mach 2001). A very tight regulation of MHC-II gene expression is thus crucial for the control of the immune response. This is particularly important in atherogenesis, which is characterized by overreactive immune responses. Down-regulation of MHC-II molecules by DHA has never been reported at the vascular level before but the group of Hughes reported similar effects in isolated human monocytes exposed to LC- $\omega 3$ PUFAs (Hughes and Pinder 2000), and several animal studies have shown that LC- 03 PUFAs can inhibit the expression of Ia molecules, the murine equivalent of human MHC-II molecules, at the surface of isolated macrophages (Kelley et al. 1985; Mosquera et al. 1990; Huang et al. 1992). The inhibition of antigen-presenting function is not expected in host defense but it could be beneficial during atherogenesis by contributing to the resolution of inflammation within the lesion. It should be noticed that wild-type control mice were not included in the present study. It is therefore possible that some of the observed effects are dependent on the antiatherogenic effects of DHA and would probably not be present in wild-type control mice. However, these latter mice do not develop atherosclerosis and the associated inflammatory processes under any circumstance or diets.

Macrophages can represent up to $60 \%$ of atheromata mass (Pello et al. 2011), and the importance of macrophage phenotype modulation in atherosclerosis is now well recognized (Mantovani et al. 2009). Indeed, functional heterogeneity and plasticity is a hallmark of macrophages that can have both pro- and anti-inflammatory properties, as they orchestrate the initiation but also the resolution phases of inflammation. Both M1-pro-inflammatory and M2-reperative macrophages have been identified within atherosclerotic lesion (Bouhlel et al. 2007), whereas it is important to keep in mind that these two extreme phenotypes as well as intermediate phenotypes and their associated biomarkers can be detected simultaneously in vivo. Interestingly, the present transcriptomic 


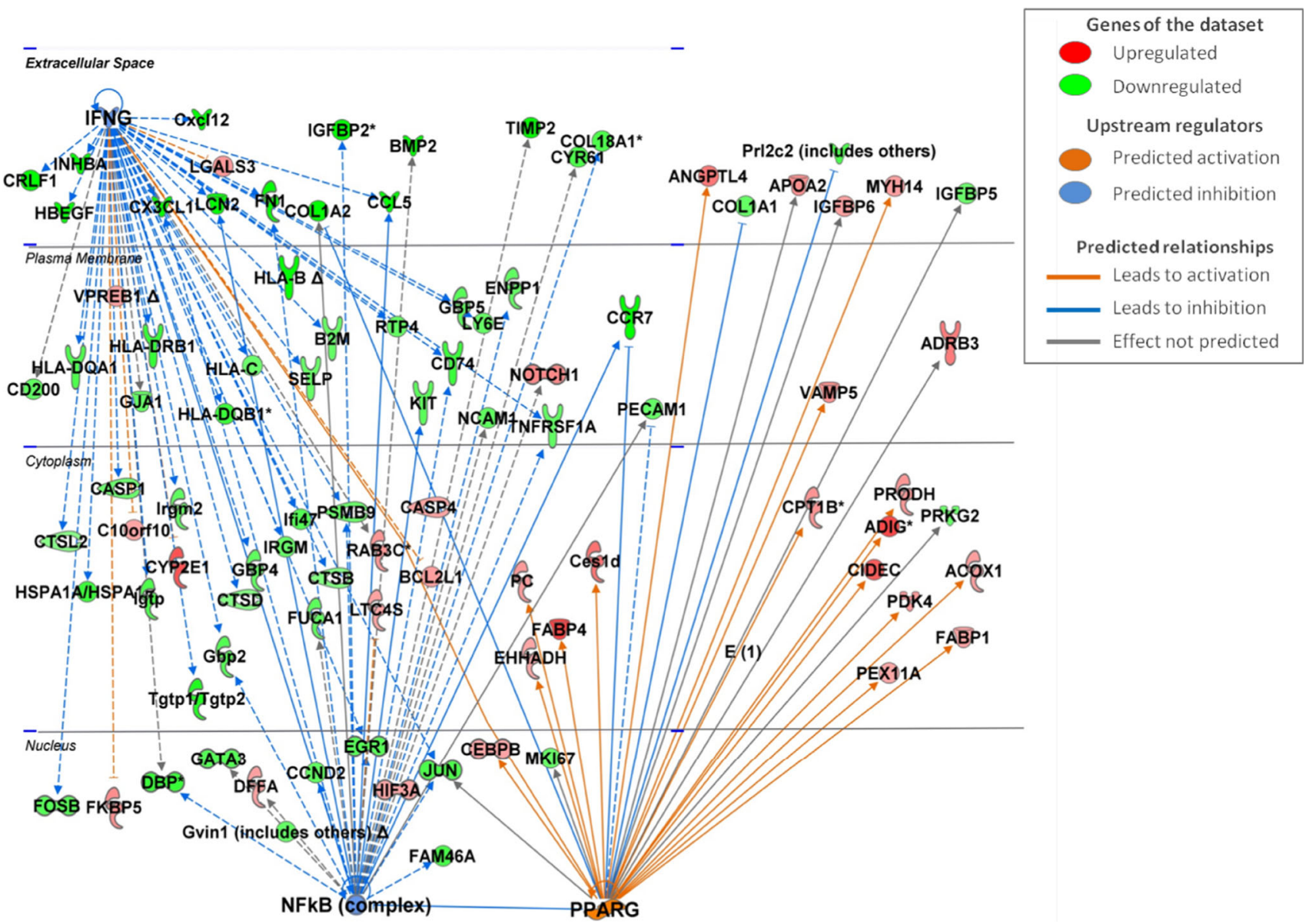

Fig. 4 Network diagram showing the genes of the dataset identified as target genes of PPAR $\gamma$, INF $\gamma$, and/or NFKB

analysis of aortic gene expression together with the analysis of macrophage phenotype within aortic root suggests that DHA supplementation may induce a preferential orientation of plaque macrophage toward a M2-like reparative phenotype. Indeed, aorta of DHA-supplemented mice showed a reduced mRNA abundance of several recognized biomarkers of M1 pro-inflammatory macrophages (i.e., CCL5, CCR7, and molecules of the MHC-II as well as LCN2) and an increased mRNA abundance of two M2 biomarkers, namely FABP4 and F13A1 (Mantovani et al. 2004; Martinez et al. 2006; Benoit et al. 2008). These genomic results are consistent with the higher abundance of Arginase I protein isoform, a specific biomarker of M2 murine macrophages (Khallou-Laschet et al. 2010). Moreover, the upstream regulators the most significantly associated with our dataset included PPAR $\gamma$, INF $\gamma$ as well as NFKB. These are three main transcriptional regulators of macrophage polarization (Lawrence and Natoli 2011) involved in the regulation of expression of most biomarkers reported above. Finally, our results obtained at the vascular level are consistent with the previous findings reported within adipose tissue macrophages showing a switch in macrophage polarization toward an M2-like phenotype in mice supplemented with DHA (Oh et al. 2010; Titos et al. 2011). Interestingly, resolvin D1, a lipid mediator arising from the lipooxigenase-dependent oxidation of DHA (Serhan et al. 2008), was suggested to be a key player of this polarization switch (Titos et al. 2011). The study of Oh et al. also suggested that the effect of DHA on macrophage polarization could be mediated by the G-protein-coupled receptor GPR120 (Oh et al. 2010).

In conclusion, the findings of the present study show that oral supplementation with DHA during atherogenesis is associated with substantial modulation of gene expression at the vascular level. These highlighted a main influence of DHA on the pathways related to inflammation and innate immunity, which could be mediated by a preferential orientation of plaque macrophages toward a M2-like reparative phenotype. These new data provide a more thorough 
a
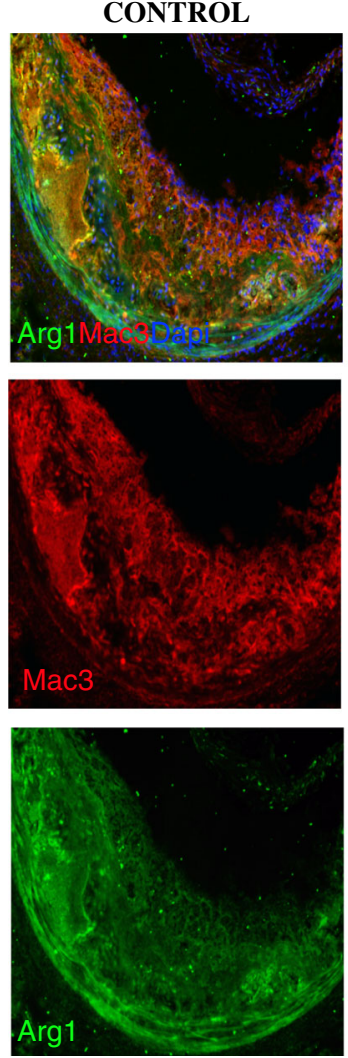

DHA
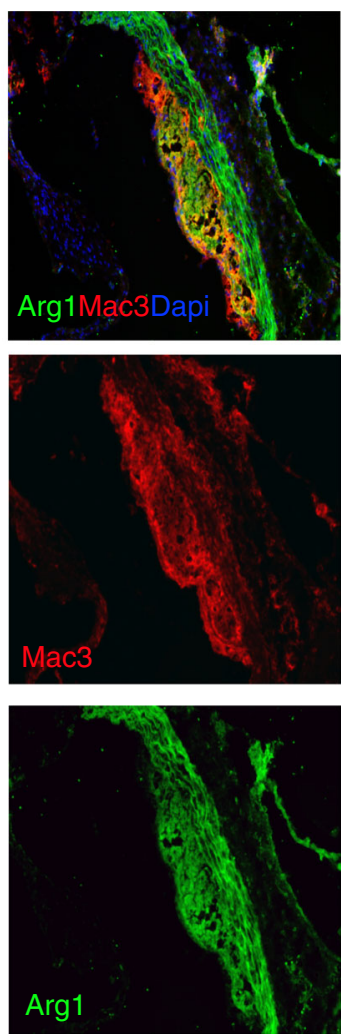

b

MAC 3

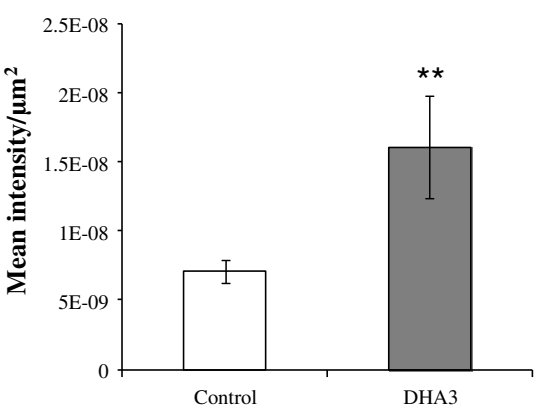

c

Arginase 1

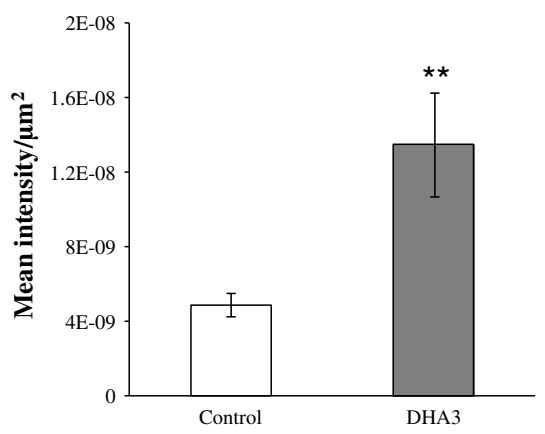

Fig. 5 Immunohistological analysis of aortic lesions regarding macrophage infiltration and phenotype. a Immunofluorescence of atherosclerotic lesions from control and DHA mice $(\times 20)$. M $\varnothing$ was identified as $\mathrm{Mac}^{+}{ }^{+}$(red) cells, and type M2 reparative macrophages were identified as Arginase $1^{+}$(green). The above 2 images represent merge images overlaying Arginase 1/Mac3/DAPI. Lesion surface was delimited on each image based on the staining of Mac3 using the ROI Manager tool in the ImageJ free software (http://rsb.info.nih.gov/ij/).

understanding of the mechanisms of action of DHA at the vascular level and could help to improve nutritional prevention of atherosclerosis and the associated cardiovascular disease.

Acknowledgments The authors acknowledge Anne de la Foye for her precious help regarding microarray hybridization design and the normalization of gene expression data. We also thank Céline Boby for her helpful advices and support during the microarray experiment and the image analysis as well as her availability. Finally, thanks to Dominique Bayle for the PCR validations and Séverine Thien for making histological sections. The transcriptomic analyses were conducted at the INRA Clermont/Theix Research Center "Metabolism Exploration Platform: from genes to metabolites." The project was supported by intramural French National Institute for Agricultural Research (INRA, http://www.alimh.inra.fr/).

Conflict of interest The authors have declared no conflict of interest.

Ethical standard This study was carried out in strict accordance with the Institutional Ethics Committee of the INRA. The protocol was approved by the Committee on the Ethics of Animal Experiments
The intensity of each staining is relative to the lesion surface; b Intensity of staining of macrophages (Mac3) relative to lesion surface in control and DHA-supplemented mice; c Intensity of staining of type M2 macrophages (ArgI) relative to lesion surface in control and DHA-supplemented mice. Student's $t$ tests were performed to compare the intensity of staining relative to the lesion surface between the control $(n=8,3$ random fields/plaque) and the DHA mice $(n=8,3$ random fields/section), $* * p<0.01$

of the Auvergne Region (Permit Number: CE-2910). All efforts were made to minimize mice suffering during the protocol and before the final experiment.

\section{References}

Benoit M, Desnues B, Mege JL (2008) Macrophage polarization in bacterial infections. J Immunol 181(6):3733-3739

Bouhlel MA, Derudas B, Rigamonti E, Dievart R, Brozek J, Haulon S, Zawadzki C, Jude B, Torpier G, Marx N, Staels B, ChinettiGbaguidi G (2007) PPARgamma activation primes human monocytes into alternative M2 macrophages with anti-inflammatory properties. Cell Metab 6(2):137-143

Calder PC (2012) The role of marine omega-3 (n-3) fatty acids in inflammatory processes, atherosclerosis and plaque stability. Mol Nutr Food Res 56(7):1073-1080

Calder PC (2013) Omega-3 polyunsaturated fatty acids and inflammatory processes: nutrition or pharmacology? Br J Clin Pharmacol 75(3):645-662

Collie-Duguid ES, Wahle KW (1996) Inhibitory effect of fish oil N-3 polyunsaturated fatty acids on the expression of endothelial cell 
adhesion molecules. Biochem Biophys Res Commun 220(3): 969-974

Dawson K, Zhao L, Adkins Y, Vemuri M, Rodriguez RL, Gregg JP, Kelley DS, Hwang DH (2012) Modulation of blood cell gene expression by DHA supplementation in hypertriglyceridemic men. J Nutr Biochem 23(6):616-621

De Caterina R, Zampolli A (2006) Antiatherogenic effects of n-3 fatty acids: evidence and mechanisms. Heart Int 2(3-4):141

De Caterina R, Cybulsky MI, Clinton SK, Gimbrone MA, Libby P Jr (1994) The omega-3 fatty acid docosahexaenoate reduces cytokine-induced expression of proatherogenic and proinflammatory proteins in human endothelial cells. Arterioscler Thromb 14(11):1829-1836

Eriksson EE (2004) Mechanisms of leukocyte recruitment to atherosclerotic lesions: future prospects. Curr Opin Lipidol 15(5): $553-558$

Forster R, Schubel A, Breitfeld D, Kremmer E, Renner-Muller I, Wolf E, Lipp M (1999) CCR7 coordinates the primary immune response by establishing functional microenvironments in secondary lymphoid organs. Cell 99(1):23-33

Galkina E, Ley K (2007) Vascular adhesion molecules in atherosclerosis. Arterioscler Thromb Vasc Biol 27(11):2292-2301

Gladine C, Roy NC, Rigaudiere JP, Laillet B, Da Silva G, Joly C, PujosGuillot E, Morio B, Feillet-Coudray C, McNabb WC, Chardigny JM, Comte B (2012) Increasing intake of long-chain n-3 PUFA enhances lipoperoxidation and modulates hepatic gene expression in a dose-dependent manner. Br J Nutr 107(9):1254-1273

Gladine C, Newman JW, Durand T, Pedersen TL, Galano JM, Demougeot C, Berdeaux O, Pujos-Guillot E, Mazur A, Comte B (2014) Lipid profiling following intake of the omega 3 fatty acid DHA identifies the peroxidized metabolites F4-neuroprostanes as the best predictors of atherosclerosis prevention. PLoS ONE 9(2):e89393

Huang SC, Misfeldt ML, Fritsche KL (1992) Dietary fat influences Ia antigen expression and immune cell populations in the murine peritoneum and spleen. J Nutr 122(6):1219-1231

Hughes DA, Pinder AC (2000) n-3 polyunsaturated fatty acids inhibit the antigen-presenting function of human monocytes. Am J Clin Nutr 71(1 Suppl):357S-360S

Hughes DA, Southon S, Pinder AC (1996) (n-3) Polyunsaturated fatty acids modulate the expression of functionally associated molecules on human monocytes in vitro. J Nutr 126(3):603-610

Huo Y, Hafezi-Moghadam A, Ley K (2000) Role of vascular cell adhesion molecule-1 and fibronectin connecting segment- 1 in monocyte rolling and adhesion on early atherosclerotic lesions. Circ Res 87(2):153-159

Ishibashi S, Goldstein JL, Brown MS, Herz J, Burns DK (1994) Massive xanthomatosis and atherosclerosis in cholesterol-fed low density lipoprotein receptor-negative mice. J Clin Invest 93(5): 1885-1893

Ishikado A, Morino K, Nishio Y, Nakagawa F, Mukose A, Sono Y, Yoshioka N, Kondo K, Sekine O, Yoshizaki T, Ugi S, Uzu T, Kawai H, Makino T, Okamura T, Yamamoto M, Kashiwagi A, Maegawa H (2013) 4-Hydroxy hexenal derived from docosahexaenoic acid protects endothelial cells via Nrf2 activation. PLoS ONE 8(7):e69415

Jaffe EA, Ruggiero JT, Falcone DJ (1985) Monocytes and macrophages synthesize and secrete thrombospondin. Blood 65(1): $79-84$

Jump DB, Depner CM, Tripathy S (2012) Omega-3 fatty acid supplementation and cardiovascular disease. J Lipid Res 53(12):2525-2545

Kelley VE, Ferretti A, Izui S, Strom TB (1985) A fish oil diet rich in eicosapentaenoic acid reduces cyclooxygenase metabolites, and suppresses lupus in MRL-lpr mice. J Immunol 134(3): 1914-1919
Keophiphath M, Rouault C, Divoux A, Clement K, Lacasa D (2010) CCL5 promotes macrophage recruitment and survival in human adipose tissue. Arterioscler Thromb Vasc Biol 30(1):39-45

Khallou-Laschet J, Varthaman A, Fornasa G, Compain C, Gaston AT, Clement M, Dussiot M, Levillain O, Graff-Dubois S, Nicoletti A, Caligiuri G (2010) Macrophage plasticity in experimental atherosclerosis. PLoS ONE 5(1):e8852

Knoch B, Barnett MP, Zhu S, Park ZA, Nones K, Dommels YE, Knowles SO, McNabb WC, Roy NC (2009) Genome-wide analysis of dietary eicosapentaenoic acid- and oleic acid-induced modulation of colon inflammation in interleukin-10 gene-deficient mice. J Nutrigenet Nutrigenomics 2(1):9-28

Lawrence T, Natoli G (2011) Transcriptional regulation of macrophage polarization: enabling diversity with identity. Nat Rev Immunol 11(11):750-761

Livak KJ, Schmittgen TD (2001) Analysis of relative gene expression data using real-time quantitative PCR and the 2(-Delta Delta C(T)) Method. Methods 25(4):402-408

Luchtefeld M, Grothusen C, Gagalick A, Jagavelu K, Schuett H, Tietge UJ, Pabst O, Grote K, Drexler H, Forster R, Schieffer B (2010) Chemokine receptor 7 knockout attenuates atherosclerotic plaque development. Circulation 122(16):1621-1628

Mantovani A, Sica A, Sozzani S, Allavena P, Vecchi A, Locati M (2004) The chemokine system in diverse forms of macrophage activation and polarization. Trends Immunol 25(12):677-686

Mantovani A, Garlanda C, Locati M (2009) Macrophage diversity and polarization in atherosclerosis: a question of balance. Arterioscler Thromb Vasc Biol 29(10):1419-1423

Martinez FO, Gordon S, Locati M, Mantovani A (2006) Transcriptional profiling of the human monocyte-to-macrophage differentiation and polarization: new molecules and patterns of gene expression. J Immunol 177(10):7303-7311

Matsumoto M, Sata M, Fukuda D, Tanaka K, Soma M, Hirata Y, Nagai R (2008) Orally administered eicosapentaenoic acid reduces and stabilizes atherosclerotic lesions in ApoE-deficient mice. Atherosclerosis 197(2):524-533

Miles EA, Wallace FA, Calder PC (2000) Dietary fish oil reduces intercellular adhesion molecule 1 and scavenger receptor expression on murine macrophages. Atherosclerosis 152(1):43-50

Moore KJ, Sheedy FJ, Fisher EA (2013) Macrophages in atherosclerosis: a dynamic balance. Nat Rev Immunol 13(10):709-721

Mosquera J, Rodriguez-Iturbe B, Parra G (1990) Fish oil dietary supplementation reduces Ia expression in rat and mouse peritoneal macrophages. Clin Immunol Immunopathol 56(1): 124-129

Oh DY, Talukdar S, Bae EJ, Imamura T, Morinaga H, Fan W, Li P, Lu WJ, Watkins SM, Olefsky JM (2010) GPR120 is an omega-3 fatty acid receptor mediating potent anti-inflammatory and insulin-sensitizing effects. Cell 142(5):687-698

Pello OM, Silvestre C, De Pizzol M, Andres V (2011) A glimpse on the phenomenon of macrophage polarization during atherosclerosis. Immunobiology 216(11):1172-1176

Reith W, Mach B (2001) The bare lymphocyte syndrome and the regulation of MHC expression. Annu Rev Immunol 19:331-373

Serhan CN, Chiang N, Van Dyke TE (2008) Resolving inflammation: dual anti-inflammatory and pro-resolution lipid mediators. Nat Rev Immunol 8(5):349-361

Shalhoub J, Falck-Hansen MA, Davies AH, Monaco C (2011) Innate immunity and monocyte-macrophage activation in atherosclerosis. J Inflamm (Lond) 8:9

Titos E, Rius B, Gonzalez-Periz A, Lopez-Vicario C, Moran-Salvador E, Martinez-Clemente M, Arroyo V, Claria J (2011) Resolvin D1 and its precursor docosahexaenoic acid promote resolution of adipose tissue inflammation by eliciting macrophage polarization toward an M2-like phenotype. J Immunol 187(10): $5408-5418$ 
Tsukamoto Y, Helsel WE, Wahl SM (1981) Macrophage production of fibronectin, a chemoattractant for fibroblasts. J Immunol 127(2):673-678

Wan W, Lionakis MS, Liu Q, Roffe E, Murphy PM (2013) Genetic deletion of chemokine receptor Ccr7 exacerbates atherogenesis in ApoE-deficient mice. Cardiovasc Res 97(3):580-588

Weber C, Erl W, Pietsch A, Danesch U, Weber PC (1995) Docosahexaenoic acid selectively attenuates induction of vascular cell adhesion molecule- 1 and subsequent monocytic cell adhesion to human endothelial cells stimulated by tumor necrosis factor-alpha. Arterioscler Thromb Vasc Biol 15(5):622-628

Zernecke A, Shagdarsuren E, Weber C (2008) Chemokines in atherosclerosis: an update. Arterioscler Thromb Vasc Biol 28(11):1897-1908 